\title{
The Impact of Timing Synchronization Errors on the Performance of OFDM/FBMC Systems
}

\author{
Yahia Medjahdi, Michel Terré, Didier Le Ruyet, Daniel Roviras and Ali Dziri \\ Electronics and Communications Laboratory, CNAM, Paris, France \\ \{yahia.medjahdi, michel.terre, leruyet, daniel.roviras, dziri\}@cnam.fr
}

\begin{abstract}
Interference at the radio receiver is a key source of degradation in quality of service of wireless communication systems. In this paper, we address the impact of imperfect intercell synchronization on the performance of orthogonal frequency division multiplexing (OFDM) and filter bank based multicarrier (FBMC) multi-cellular networks. Based on computing the moment generating functions of the asynchronous interference power, a useful analytical method for computing the average error rates is derived considering the frequency correlation fading between adjacent interfering subcarriers.

Index Terms-Inter-channel Interference, OFDM, FBMC, asynchronous, selective frequency channel, moment generating function (MGF), correlated Rayleigh fading.
\end{abstract}

\section{INTRODUCTION}

Multicarrier systems are widely used today due to their robustness to multipath effects and efficient implementation using FFT. An orthogonal Frequency Division Multiplexing (OFDM) system is a type of multicarrier modulation which consists of splitting up a wide band signal at a high symbol rate into several lower rate signals, each one occupying a narrower band. System performance improves because subcarriers experience flat fading channels and are orthogonal to one another thus minimizing the threat of interference. However, the OFDM performance tends to suffer from degradation because of possible episodes of imperfect time and frequency synchronization, since a loss in orthogonality can occur between subcarriers at the OFDM receiver [1].

The impact of asynchronous interference in OFDM systems has been intensively investigated in the literature. Using the Gaussian approximation for interchannel interference, [2] gives approximate expressions of the symbol error rates. Moreover, the degradation of the signal to interference plus noise ratio is a common criterion to analyze the impact of timing non-synchronization on the system performance [1]. In [3], an interference modeling, based on the so called Interference Table [4], has been developed for two multicarrier techniques: CP-OFDM with a rectangular pulse shape and for Filter Bank based Multi-Carrier (FBMC) with a prototype filter designed for a better frequency selectivity using the frequency sampling technique [5].

Although interference analysis in OFDM single user has become popular in literature e.g [6], [7], the extension of this

${ }^{1}$ Part of this work has been supported by PHYDYAS UE project (FP7-ICT2007-1-211887) analysis to a multi-cellular environment is not so straightforward. This problem is significant for the following reasons. First, in a multi-cellular environment the interference stems from subcarriers distributed among several transmitters which require more than one random variable (RV) to model this interference, therefore, the analysis becomes more difficult. Second, in contrast to many researches based on the classical Gaussian approximation [2], [8], we cannot always rely on this approximation. For example, when the number of interferers is large but there are dominant interferers, the central limit theorem is no longer applicable [9].

This paper presents an analytical interference analysis related to asynchronous downlink OFDM/FBMC in multicellular environment, providing an exact expression of average error rates in frequency selective fading channels.

Based on the interference table model introduced in [3], we derive an explicit form of the bit error rate of timeasynchronous OFDM and FBMC systems in the case of the block subcarrier assignment taking into account the correlation between the subchannel gains belonging to a given block subcarrier. The computation of the average error rate is based on the moment generating function of the interference power.

The rest of the paper is organized as follows. Section II is devoted to describing the system model of the downlink of OFDM and FBMC based multi-cellular networks. A brief review of interference table modeling is given in Section III. We further derive an explicit expression of the average error rates of asynchronous OFDM/FBMC systems in Section IV. Simulation results are presented and discussed in Section V. Section VI concludes the paper.

\section{The System MODEL}

We consider the downlink transmission in OFDM/FBMC based multi-cellular networks depicted in Fig. 1 (a). The reference mobile user is located at $(u, v)$ in the hexagonal coordinate system. The reference base station is assumed to be situated at the origin $\left(u_{0}, v_{0}\right)=(0,0)$. In this analysis, we consider two tiers of the neighboring cells that are surrounding the reference mobile user. Let the $k$-th base station be located at $\left(u_{k}, v_{k}\right)$, then, the distance between the reference mobile user and the $k$-th base station is given by

$$
d_{k}=\sqrt{\left(u_{k}-u\right)^{2}+\left(v_{k}-v\right)^{2}+\left(u_{k}-u\right)\left(v_{k}-v\right)}
$$

The cell radius is denoted by $R$ in Fig. 1 . 


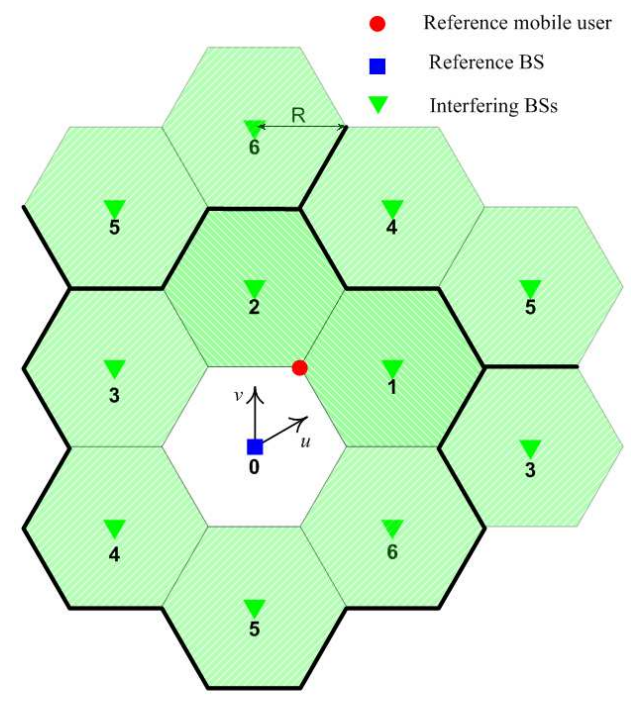

(a)

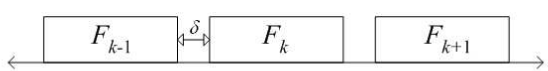

(b)

Fig. 1. (a). The downlink of OFDM/FBMC based networks (b). The subcarrier assignment scheme

Concerning the frequency reuse scheme, the subcarriers are allocated according to the most common subcarrier assignment scheme, namely, the block subcarrier assignment scheme which is described in Fig. 1 (b). We assume in this scheme that $\delta$ adjacent subcarriers to each block are free and serve as guard bands between the different blocks. It should be noticed that the frequency reuse factor is $1 / 7$.

The reference mobile user is assumed to be perfectly synchronized with its base station but it is not necessarily synchronized with the other base stations. We can express the composite signal at the reference receiver by the sum of the desired signal coming from the reference base station and the interference signal coming from the surrounding base stations,

$$
\begin{aligned}
& r(t)=\underbrace{d_{0}^{-\beta / 2} s_{0}(t) * h_{0}(t)}_{\text {desired signal }} \\
& \quad+\underbrace{\sum_{k=1}^{K} d_{k}^{-\beta / 2} s_{k}\left(t-\tau_{k}\right) * h_{k}(t)}_{\text {interference signal }}+n(t)
\end{aligned}
$$

where

- $K$ is the total number of neighboring cells

- $s_{k}(t)$ is the transmitted signal from the $k$-th base station

- $\tau_{k}$ and $h_{k}(t)$ denote respectively the timing offset and the impulse response of the channel between the reference mobile user and the $k$-th base station

- $n(t)$ is the additive white Gaussian noise (AWGN)

- $\beta$ is the path loss exponent

Because of the timing misalignment between the neighboring cells and the reference one, the signals arriving from the cells in the vicinity will appear non-orthogonal to the desired

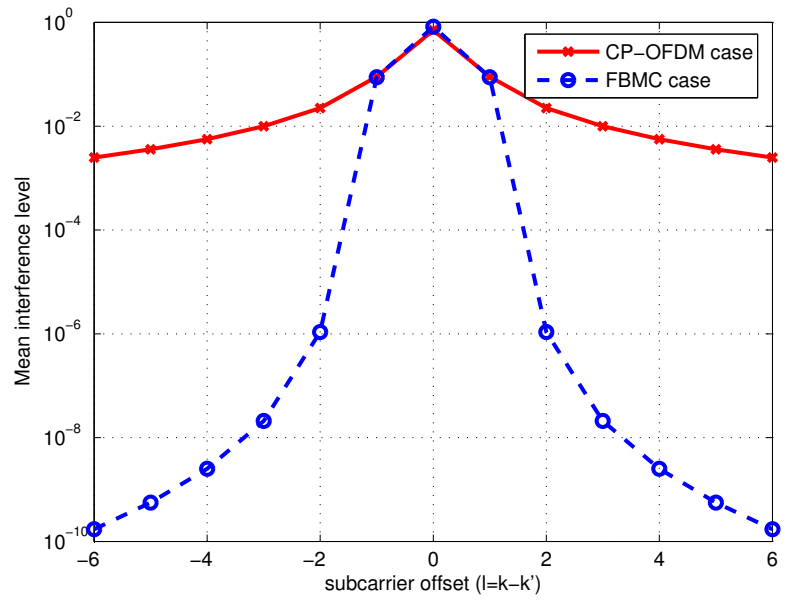

Fig. 2. The mean interference level in CP-OFDM $(\Delta=T / 8)$ and FBMC (PHYDYAS prototype filter, $K=4$ )

signal. This non-orthogonality will generate interference and will degrade the SINR. This degradation will be investigated in the next section.

\section{INTERFERENCE AND SINR ANALYSIS}

In this section we present an accurate interference analysis that considers the multipath effects on the different signals and also the timing offsets between the interfering base stations and the reference one. Direct analytical methods lead to complex integral expressions that require huge computational efforts. We present here an attractive non-direct analytical method that significantly reduces the complexity of the analysis. This method is based on the interference table model proposed in [4].

\section{A. Interference Tables}

In [4], OFDM/FBMC interference tables are given. These tables model the correlation between subcarriers caused by the timing misalignment between the different transmitters (base stations in our analysis). In Fig. 2, the mean interference is plotted against the subcarrier space between the interfering subcarrier and the target one. It is worth noticing that this interference has been computed considering CP-OFDM system with a CP duration $\Delta=T / 8$, where $T$ is the OFDM symbol duration and FBMC system using the PHYDYAS prototype filter [5] with an overlapping factor of 4 .

\section{$B$. Interference power in a selective frequency channel}

It has been demonstrated in [3], that the asynchronous interference power arriving through a selective frequency channel can be calculated using the following expression

$$
P_{\text {interf }}(m, \tau)=d^{-\beta} P_{\text {trans }}\left(m^{\prime}\right) I\left(\tau,\left|m^{\prime}-m\right|\right)\left|H\left(m^{\prime}\right)\right|^{2}
$$

where

- $d$ is the distance between the interferer and the victim user

- $P_{\text {trans }}\left(m^{\prime}\right)$ is the transmitted power on the $m^{\prime}$-th interfering subchannel 
- $I\left(\tau,\left|m^{\prime}-m\right|\right)$ is the interference table coefficient corresponding to a timing offset $\tau$ and $m$ denotes the index of the victim subchannel

- $\left|H\left(m^{\prime}\right)\right|^{2}$ is the power channel gain between the interfering transmitter and the reference receiver on the $m^{\prime}$-th subchannel

In the multi-cell case described in Section II, the interference is caused by the $K$ base stations surrounding the reference cell. We can easily express the total interference power occurring at the output filter of the reference mobile user by

$$
\begin{aligned}
& P_{\text {interf }}\left(m,\left\{\tau_{k}, k=1, \ldots, K\right\}\right)= \\
& \sum_{k=1}^{K} \sum_{m^{\prime} \in F_{k}} d_{k}^{-\beta} P_{\text {trans }}\left(m^{\prime}\right) I\left(\tau_{k},\left|m^{\prime}-m\right|\right)\left|H_{k}\left(m^{\prime}\right)\right|^{2}
\end{aligned}
$$

where $F_{k}$ denotes the set of subcarriers that are assigned to the $k$-th base station. We recall that $\tau_{k}$ and $\left|H_{k}\left(m^{\prime}\right)\right|^{2}$ are respectively the timing offset and the power channel gain between the reference mobile user and the $k$-th base station.

As aforementioned, the reference mobile user is assumed to be perfectly synchronized with its base station. Consequently, the power of the desired signal can be written as

$$
P_{\text {desired }}(m)=d_{0}^{-\beta} P_{\text {trans }}(m)\left|H_{0}(m)\right|^{2}
$$

According to (4) and (5), the SINR is given by

$$
\operatorname{SINR}(m)=\frac{\left|H_{0}(m)\right|^{2}}{\sum_{k=1}^{K} \sum_{m^{\prime} \in F_{k}} A_{k, m, m^{\prime}}\left|H_{k}\left(m^{\prime}\right)\right|^{2}+b}
$$

where $N_{0}$ denotes the noise power spectral density and $B_{s c}$ is the bandwidth of the $m$-th subchannel. where

$$
\begin{gathered}
A_{k, m, m^{\prime}}=\left[\frac{d_{k}}{d_{0}}\right]^{-\beta} \frac{P_{\text {trans }}\left(m^{\prime}\right)}{P_{\text {trans }}(m)} I\left(\tau_{k},\left|m^{\prime}-m\right|\right) \\
b=\frac{N_{0} B_{s c}}{d_{0}^{-\beta} P_{\text {trans }}(m)}
\end{gathered}
$$

\section{Average Error Rates Analysis}

In the following derivation we analyze the QPSK constellation case, the extension to another MQAM constellation is straightforward. The calculation of the bit error rate of this constellation is readily available in the literature when the decision variables are Gaussian random variables [11]

$$
\operatorname{BER}(\mathrm{SNR})=\frac{1}{2} \operatorname{erfc}\left(\sqrt{\frac{1}{2} \mathrm{SNR}}\right)
$$

Therefore, by conditioning on the set of variables $\left\{H_{0}(m), H_{k}\left(m^{\prime}\right), \forall k, m, m^{\prime}\right\}$ and substituting (6) in (9), we can obtain the exact closed form for the conditional error probabilities in the presence of interference (10).

$$
\begin{aligned}
& \left.\operatorname{BER}(\mathrm{SNR})\right|_{H_{0}(m), H_{k}\left(m^{\prime}\right)}= \\
& \frac{1}{2} \operatorname{erfc}\left(\frac{1}{2} \frac{\left|H_{0}(m)\right|^{2}}{\sum_{k=1}^{K} \sum_{m^{\prime} \in F_{k}} A_{k, m, m^{\prime}}\left|H_{k}\left(m^{\prime}\right)\right|^{2}+b}\right)^{1 / 2}
\end{aligned}
$$

In order to reduce the complexity of computing the average bit error rate which requires $K \times N$ integrations into only one integration, we refer to the following lemma [10], which is based on the moment generating function of the interference power.

Lemma: Let $x$ be a unit-mean gamma random variable (RV) with parameter $\alpha$, and let $y$ be an arbitrary non-negative random variable that is independent of $x$. Then

$$
\begin{aligned}
E_{x, y} & {\left[\operatorname{erfc}\left(\frac{x}{y+b}\right)^{1 / 2}\right]=1-\frac{2}{\pi} \frac{\Gamma\left(\alpha+\frac{1}{2}\right)}{\Gamma(\alpha)} } \\
& \times \int_{0}^{+\infty} \frac{e^{-z}}{\sqrt{z}}{ }_{1} F_{1}\left(1-\alpha ; \frac{3}{2} ; z\right) \mathcal{M}_{y}(\alpha z) e^{-z \alpha b} d z
\end{aligned}
$$

where ${ }_{1} F_{1}(; ;)$ is the confluent hypergeometric function of the first kind [12] and $\mathcal{M}_{y}(z)=E_{y}\left[e^{-z y}\right]$ is the moment generating function (MGF) of $y$.

As $\left|H_{0}(m)\right|$ is a Rayleigh random variable, $x=\left|H_{0}(m)\right|^{2}$ is an exponential $\mathrm{RV}$ with a probability density function (pdf) $f(x)=e^{-x}$. In other words, $x$ is a unit-mean gamma RV with $\alpha=1$. Since ${ }_{1} F_{1}(0 ; 3 / 2 ; z)=1$, the expression (11) becomes

$$
E_{x, y}\left[\operatorname{erfc}\left(\frac{x}{y+b}\right)^{1 / 2}\right]=1-\frac{1}{\sqrt{\pi}} \int_{0}^{+\infty} \frac{e^{-z(1+b)}}{\sqrt{z}} \mathcal{M}_{y}(z) d z
$$

In our analysis, the random variable related to the total interference power is defined by

$$
y=2 \sum_{k=1}^{K} \sum_{m^{\prime} \in F_{k}} A_{k, m, m^{\prime}} y_{k, m^{\prime}}
$$

where $y_{k, m^{\prime}}=\left|H_{k}\left(m^{\prime}\right)\right|^{2}$.

As the signals coming from the different interfering cells $\left\{\sum_{m^{\prime} \in F_{k}} A_{k, m, m^{\prime}} y_{k, m^{\prime}}, \forall k\right\}$ are independent, the moment generating function of $y$ is given by

$$
\begin{aligned}
\mathcal{M}_{y}(z) & =E_{\left\{y_{k, m^{\prime}}, \forall k, m^{\prime}\right\}}\left[e^{-2 z \sum_{k=1}^{K} \sum_{m^{\prime} \in F_{k}} A_{k, m, m^{\prime}} y_{k, m^{\prime}}}\right] \\
& =\prod_{k=1}^{K} \mathcal{M}_{k}(z)
\end{aligned}
$$

where

$$
\mathcal{M}_{k}(z)=E_{\left\{y_{k, m^{\prime}}, m^{\prime} \in F_{k}\right\}}\left[e^{-2 z \sum_{m^{\prime} \in F_{k}} A_{k, m, m^{\prime}} y_{k, m^{\prime}}}\right]
$$

However, the RVs $\left\{y_{k, m^{\prime}}=\left|H_{k}\left(m^{\prime}\right)\right|^{2}, m^{\prime} \in F_{k}\right\}$ are correlated because they belong to the same cluster used by the $k$-th base station. To deal with this problem, let $\Omega_{k}$ defined by

$$
\Omega_{k}=\left[\rho_{i, j}\right]_{(i, j) \in F_{k} \times F_{k}}
$$

where $\rho_{i, j}=\rho_{j, i}$, be the square root of the variance-covariance matrix of the RVs $\left\{y_{k, m^{\prime}}, m^{\prime} \in F_{k}\right\}$. 
Following [13], the MGF $\mathcal{M}_{k}(z)$ is obtained by

$$
\mathcal{M}_{k}(z)=\left|I_{L_{k}}+2 \Omega_{k} D_{k}^{A} z\right|^{-1}
$$

where $I_{L_{k}}$ is the $L_{k} \times L_{k}$ identity matrix and $L_{k}$ denotes the cardinal of $F_{k} . D_{k}^{A}$ is a diagonal matrix of diagonal elements,

$$
D_{k}^{A}(i, i)=A_{k, m, i} \quad i \in F_{k}
$$

Substituting (16) in the expression (14), we obtain the MGF related to the total interference RV $y$ defined in (13),

$$
\mathcal{M}_{y}(z)=\prod_{k=1}^{K} \mathcal{M}_{k}(z)=\prod_{k=1}^{K}\left|I_{L_{k}}+2 \Omega_{k} D_{k}^{A} z\right|^{-1}
$$

Therefore, using the expressions (12) and (18), the final expression of the average BER for $K$ interfering base stations is shown in (19).

$$
\begin{aligned}
& \text { BER }_{\text {average }}= \\
& \frac{1}{2}-\frac{1}{2 \sqrt{\pi}} \int_{0}^{+\infty} \frac{e^{-z(1+2 b)}}{\sqrt{z}} \prod_{k=1}^{K}\left|I_{L_{k}}+2 \Omega_{k} D_{k}^{A} z\right|^{-1} d z
\end{aligned}
$$

\section{Simulation Results}

In the previous section, we have derived a closed-form expression of the average error rates in the downlink of an asynchronous $K$-cell network. In contrast to direct complex analytical methods, this expression presents an efficient approach to compute the average BER with a significantly reduced computational complexity. In this section, we present numerical results for the downlink of OFDM and FBMC systems with the block subcarrier scheme as described in Section II.

We have considered the Pedestrian-A model as a Rayleigh fading propagation channel where the parameters are given in [14]. The path loss of a received signal at a distance $d$ is governed by the following expression

$$
\Gamma_{\text {loss }}(d)=128.1+37.6 \log _{10}(d)[d B]
$$

On the other hand, we consider a system with $N=1024$ subcarriers using a total bandwidth of $10 \mathrm{MHz}$. The noise term is characterized by a thermal noise density of $-174 \mathrm{dBm} / \mathrm{Hz}$. The prefix cyclic duration is fixed at $\Delta=T / 8$, and the size of the subcarrier block is set at 18 subcarriers. For the FBMC system, we recall that we use the PHYDYAS prototype filter with an overlapping factor of 4 [5]. It is worth mentioning that the following results are compared to the perfect synchronized scenario in which the orthogonality between the different subchannels is maintained.

In Fig. 3, we investigate the accuracy of the BER expression. The average BERs of OFDM and FBMC modulations are plotted against the SNR, in absence of a guard band between the clusters of the different cells $(\delta=0)$. Both theoretical and simulation results are displayed in Fig. 3. The theoretical results are evaluated using (19). The exact theoretical results depicted in Fig. 3 show an excellent match to the corresponding simulation results. In this case, we assume that

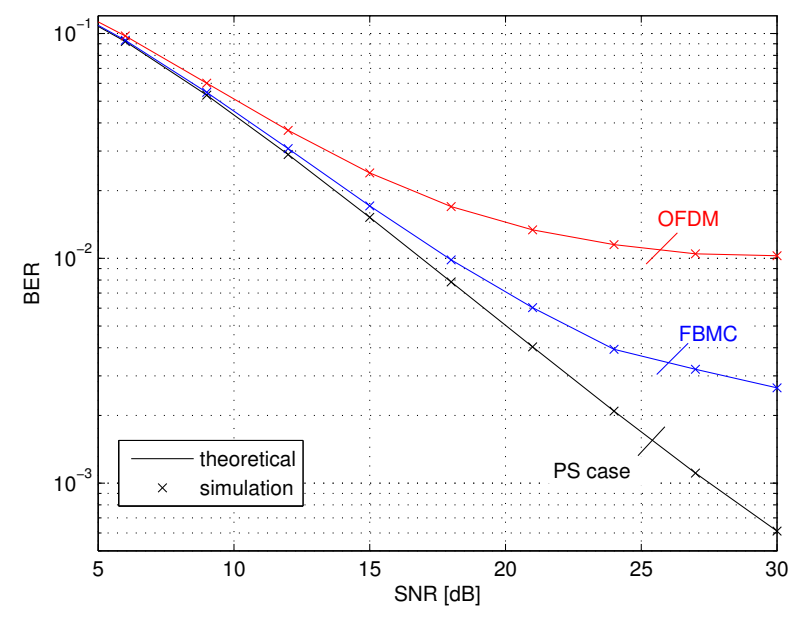

Fig. 3. The OFDM/FBMC average BER against the SNR for $\tau \in[0, \mathrm{~T}]$, the guard-band size $\delta=0$

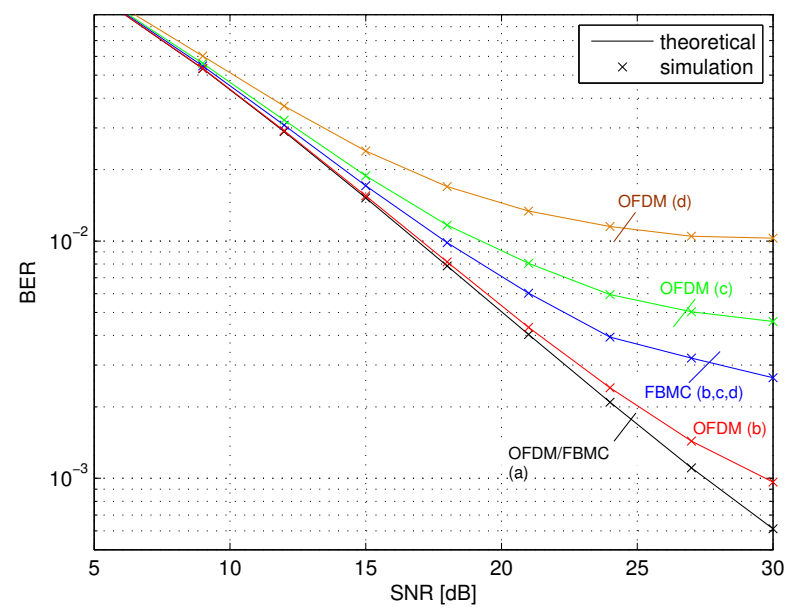

Fig. 4. The average BER for different timing offset intervals (a). PS (b). $\tau \in[0, T / 7]$ (c). $\tau \in[0, T / 4]$ (d). $\tau \in[0, T]$, the guard-band size $\delta=0$

the timing offset $\tau$ is a uniform RV in the interval [0,T]. Fig. 3 also shows that the timing synchronization errors cause a severe degradation in the average error rate. Moreover, this degradation becomes large when increasing the SNR level. We can also see an error floor at high SNR values. This observation can be explained by the fact that the noise level is negligible compared to the asynchronous interference caused by the other BSs. Such a case is expected in the interferencelimited scenarios. On the other hand, we observe a better performance of the asynchronous FBMC when compared to the asynchronous OFDM. Such a gain can be explained by the fact that only the two subcarriers on the edge suffer from the interference caused by their adjacent subcarriers in the FBMC case (see Fig. 2). However, in the OFDM case, the entire cluster suffers from the interference caused by all neighboring clusters (see Fig. 2).

In Fig. 4, we plot the average BER versus the SNR with different timing offset scenarios: the perfect synchronized case 


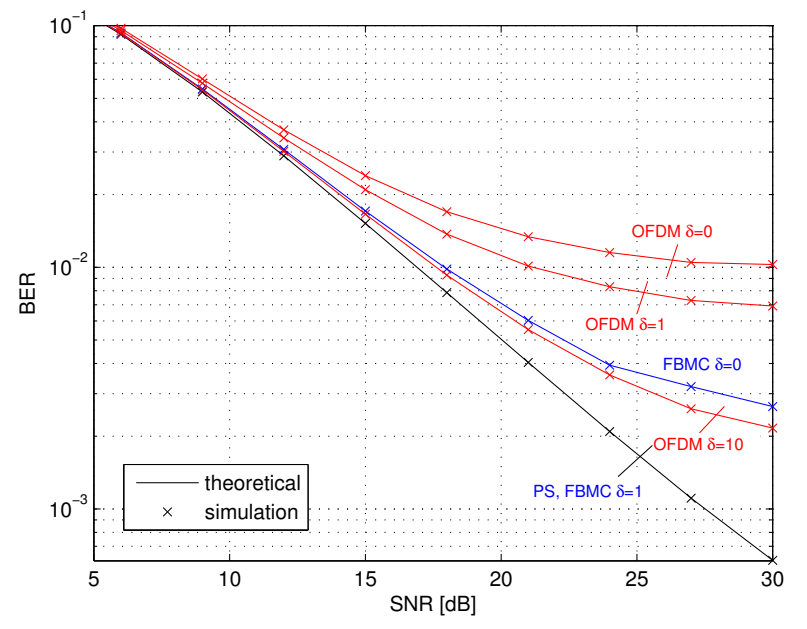

Fig. 5. The average BER for different guard-band sizes $\delta=0,1,10$, the timing offset interval $\tau \in[0, \mathrm{~T}]$

in scenario (a), $[0, T / 7]$ in scenario (b), $[0, T / 4]$ in scenario (c) and $[0, T]$ in scenario $(d)$.

In the OFDM system, the degradation is severe and increases when the timing error interval is larger. We can explain this result as follows: when the timing offset is lower than the cyclic prefix duration $\tau \in[0, \Delta]$, the orthogonality between the different clusters is maintained; otherwise the reference user will suffer from an asynchronous interference. Since the timing offset is a uniform random variable, the probability obtaining the performance of the perfect synchronized case is given by the $\mathrm{CP}$ duration over the whole timing offset interval $\left(\Delta / \tau_{\max }\right)$. The probability of the orthogonality decreases as $\tau_{\max }$ increases. Therefore, the average error probability becomes higher. On the other hand, the FBMC system is not sensitive to the timing offset interval length because the interference at the two subcarriers of the edge is roughly invariable with respect to the timing offset value (see Fig. 2).

The impact of the guard-band length $\delta$ on the system performance has also been investigated. Fig. 5 shows the OFDM and FBMC average BER against the SNR for different guard band values $\delta=0,1$ and 10 subcarriers; we assume also that the timing offset $\tau$ is a uniform RV defined on $[0, \mathrm{~T}]$. Comparing the different curves, one can see that the performance improves when increasing $\delta$. However, there is still a gap with respect to the perfect synchronized case even for a guard-band of 10 subcarriers. In contrast to the OFDM case, the FBMC waveform presents an excellent performance and provides the performance of the PS case for a guard-band of a single subcarrier $\delta=1$. This result can also be explained by referring to Fig. 2 which shows that in FBMC, the interference power does not exceed $-60 \mathrm{~dB}$ of the useful power when the interfering subcarrier is situated at two subcarriers from the victim one. It should be noticed that Fig. 4 and 5 show also an excellent match between the simulation and theoretical results obtained by the closed-form expression of the average BER given in (19).

\section{CONCLUSION}

In this paper, we have investigated the impact of timing synchronization errors on the performance of the downlink of OFDM and FBMC based multi-cellular networks. We first give a brief review of the interference table model. We then develop a theoretical derivation of the average error expression. In contrast to the direct analytical method that requires huge computational efforts, the obtained closed-form expression reduces significantly the computation complexity. The accuracy of the obtained expression has been validated through the different simulation results. A global evaluation has been performed taking into account two parameters: the timing error range, the guard band length. Through this evaluation, we have shown that in OFDM case, timing errors between BSs cause a severe degradation in system performance. This result is explained by the loss of orthogonality between all system subcarriers. In contrast to the OFDM system, the FBMC waveform is demonstrated to be less sensitive to timing errors between the different cells, due to the better frequency localization of the prototype filter.

\section{REFERENCES}

[1] Y. Mostofi and D. Cox, "Mathematical Analysis of the Impact of Timing Synchronization Errors on the Performance of an OFDM System," IEEE Trans. Commun., vol. 54, no. 2, Feb. 2006.

[2] X. Wang, T. T. Tjhung, Y. Wu, and B. Caron, "SER performance evaluation and optimization of OFDM system with residual frequency and timing offsets from imperfect synchronization," IEEE Trans. Broadcast., vol. 49, no. 2, pp. 170-177, Jun. 2003.

[3] Y. Medjahdi, M. Terré, D. Le Ruyet, D. Roviras, "Asynchronous OFDM/FBMC Interference Analysis in Selective Channels", in Proc. IEEE International Symposium on Personal, Indoor and Mobile Radio Communications, Sep. 2010, pp. 538-542.

[4] Y. Medjahdi, M. Terré, D. Le Ruyet, D. Roviras, J.A. Nossek and L. Baltar, "Inter-Cell Interference Analysis for OFDM/FBMC Systems", Proc. of IEEE-SPAWC'09 conference, Jun. 2009, pp. 598-602.

[5] M. G. Bellanger, "Specification and design of a prototype filter for filter bank based multicarrier transmission," in Proc. IEEE Int. Conf. Acoustics, Speech, and Signal Processing, May 2001, pp. 2417-2420.

[6] T.Pollet, M.Van Bladel and M. Moeneclaey "BER Sensitivity of OFDM Systems to Carrier Frequency Offset and Wiener Phase Noise," IEEE Trans. Commun., vol. 43, no. 2/3/4, pp.191-193, Feb-Apr. 1995.

[7] T.Pollet and M. Moeneclaey "The Effect of Carrier Frequency Offset on the Performance of Band limited Single Carrier and OFDM Signals," in Proc. IEEE GLOBECOM, Nov. 1996, pp 719-723.

[8] J. Cheng and N. C. Beaulieu, "Accurate DS-CDMA bit-error probability calculation in Rayleigh fading," IEEE Trans. Wireless Commun., vol. 1, no. 1, pp. 3-15, Jan. 2002.

[9] A. Giorgetti and M. Chiani, "Influence of fading on the Gaussian approximation for BPSK and QPSK with asynchronous cochannel interference," IEEE Trans. Wireless Commun., vol. 4, no. 2, pp. 384$389,2005$.

[10] K. A. Hamdi, "A Useful Technique for Interference Analysis in Nakagami Fading", IEEE Trans. Commun., vol 55, no. 6, June 2007.

[11] K. Cho and D. Yoon, "On the general BER expression of one and two dimensional amplitude modulation," IEEE Trans. Commun., vol. 50, no. 7, pp. 10741080, Jul. 2002.

[12] M. Abramowitz and I. A. Stegun, Handbook of Mathematical Functions. New York: Dover, 1965.

[13] A.S. Krishnamoorthy and M. Parthasarathy, "A multi-variate gammatype distribution", Annals of Mathematical Statistics, pp. 549-557, 1951.

[14] Rec. ITU-R M.1225 1, Guidelines for evaluation of radio transmission technologies for IMT-2000, 1997. 